\section{Diagnosis of Spinal Metastasis: Usefulness of Additional Diffusion-Weighted Imaging} 척추 전이의 진단: 확산강조 자기공명영상 추가의 유용성

\author{
Yong Ju Kim, MD ${ }^{1} \mathbb{D}$, Joon Woo Lee, $\mathrm{PhD}^{1^{*}} \mathbb{D}$, Eugene Lee, $\mathrm{PhD}^{1}$ (iD), \\ Chankue Park, MD² ${ }^{2}$, Yusuhn Kang, $\mathrm{PhD}^{1}$ (D), \\ Joong Mo Ahn, $\mathrm{PhD}^{1}$ (D), Heung Sik Kang, $\mathrm{PhD}^{1}$ \\ ${ }^{1}$ Department of Radiology, Seoul National University Bundang Hospital, Seongnam, Korea \\ ${ }^{2}$ Department of Radiology, Pusan National University College of Medicine, Pusan National University \\ Yangsan Hospital, Yangsan, Korea
}

Purpose To determine the usefulness of diffusion weighted-MRI (DW-MRI) in the evaluation of spinal metastasis.

Materials and Methods From July to August 2017, 48 whole-spine DW-MRI to detect metastasis in patients with extra-spinal tumors were retrospectively evaluated by three radiologists. The usefulness of DW-MRI was evaluated in four groups based on the change in confidence rating between two sessions: 1 (T1- and T2-weighted and contrast-enhanced images) and 2 (additional DW-MRI). The associations of the usefulness with age, sex, primary cancer, bone type with metastasis, number of probable metastatic segments in session 1 , and anatomic locations were assessed in vertebral body and posterior element cases.

Results According to the readers 1, 2, and 3, there were 18, 19, and 16 vertebral body cases, respectively, and 12,13 , and 9 posterior element cases, respectively. In the group with no excepted metastasis, DW-MRI was useful in $52-59 \%$ of vertebral body cases and $39-67 \%$ of posterior element cases. There were no significant differences in the usefulness with respect to the number of probable metastatic segments in session 1, age, sex, primary cancer, bone type with metastasis, or anatomic location.

Conclusion DW-MRI could be used to evaluate spinal metastasis. However, there were no differences in the usefulness with respect to the anatomic location.

Index terms Spine; Neoplasm Metastasis; Diffusion Magnetic Resonance Imaging
Received January 20, 2019

Revised May 8, 2019

Accepted May 17, 2019

${ }^{*}$ Corresponding author Joon Woo Lee, PhD Department of Radiology, Seoul National University Bundang Hospital, 82 Gumi-ro 173beon-gil, Bundang-gu, Seongnam 13620, Korea.

Tel 82-31-787-7619

Fax 82-31-787-4011

E-mail joonwoo2@gmail.com

This is an Open Access article distributed under the terms of the Creative Commons Attribution Non-Commercial License (https://creativecommons.org/ licenses/by-nc/4.0) which permits unrestricted non-commercial use, distribution, and reproduction in any medium, provided the original work is properly cited.

\section{ORCID iDs}

Yong Ju Kim (D) https://

orcid.org/0000-0002-4129-491X Joon Woo Lee (1) https://

orcid.org/0000-0002-7106-5229 Eugene Lee (D) https:// orcid.org/0000-0003-4205-2362 Chankue Park (D) https:// orcid.org/0000-0003-2937-114X Yusuhn Kang (D) https:// orcid.org/0000-0003-1838-2564 Joong Mo Ahn (D) https:// orcid.org/0000-0002-1157-0020 Heung Sik Kang (D) https:// orcid.org/0000-0002-7024-388X

서론

척추는 골 전이 중 가장 흔한 부위이며, 전체 전이 중에서는 폐와 간에 이어 3 번째로 흔한 부위이다( $(1,2)$. 암으로 사망하는 환자의 약 30 70\%는 사후 검사에서 척추 전이가 있었으며, 
약 $14 \%$ 의 환자는 이환 기간 동안 척추 전이로 인한 증상을 경험하게 된다(3). 척추 전이의 주요 임 상 증상은 국소적인 통증 혹은 신경근 통증이며, 운동성 약화, 감각 상실 및 괄약근 통제 상실 등의 증상을 동반하거나 동반하지 않을 수도 있다. 암 환자가 통증이나 신경 증상을 호소하는 경우 가 장 적절한 치료를 시작하기 위해 병변의 존재, 수 및 범위를 평가하기 위해 영상의학적 평가가 반 드시 선행되어야 한다.

자기공명영상(이하 MRI)은 오랫동안 척추와 척수 전이 평가를 위한 유용한 방법으로 오랫동안 인식되어 왔으며(4), 특히 척추 전이의 조기 진단을 다른 검사들보다 용이하게 하는 것으로 입증되 었다(5-7). 미국 영상의학과 의사협회(American College of Radiology; 이하 ACR)는 허리 통증이 있는 암 환자의 평가를 위해 조영 증강을 포함하지 않은 MRI를 통한 평가를 권고하였다. 그러나, 어떤 MRI 프로토콜이 척추 전이의 평가에 가장 적합한지에 대한 표준적 합의는 아직 부족한 상태 이다(8-10).

확산강조영상(diffusion weighted image; 이하 DWI)은 악성 종양의 평가에 있어서 여러 장기 에서 유용성이 입증되었다(11, 12). 또한, 척추 압박 골절에 대한 평가에서 병적 골절(pathologic fracture)과 양성 골다공증성 골절(benign osteoporotic fracture) 사이의 감별에 DWI가 도움이 된다는 보고가 있었고(13), 전신의 골 전이 평가에 기존의 MRI에 DWI를 추가하였을 때 도움이 된 다는 보고도 있었다(14). 또한, 척추 전이 병변에 대한 치료 반응의 평가에 DWI가 도움이 된다는 보고도 있었다(15).

본 연구에서는 척추 전이 여부의 평가에서 $\mathrm{DWI}$ 의 유용성에 대해 평가하는 것을 목표로 하였다.

\section{대상과 방법}

본 연구는 본원 연구윤리위원회(Institutional Review Board)의 승인 하에 진행되었고, 후향적 연구로 정보 제공자 동의서는 면제되었다(IRB No. B-1806-472-107).

2017년 7월부터 8월 사이에 본원에서 전이암 진단 및 평가를 위하여 척추 MRI를 촬영한 환자들 중 DWI를 포함한 환자는 총 64 명이었다. 이들 중 암 환자로 확진되지 않은 환자 5 명을 제외하였 고, 척추 혹은 척수의 원발 암으로 확진된 환자 7명을 제외하였고, 척추 전이 여부를 평가하기 위 한 목적이 아닌 환자들 4명을 제외하였다(Fig. 1). 임상 소견은 전자의무기록을 참고하였으며 나 이, 성별, 원발 종양에 대해 검토하였다.

최종적으로 31 명의 남성(평균 연령: 62.2세, 연령 분포: 10 83세)과 17 명의 여성(평균 연령: 60.5세, 연령 분포: 11 87세)의 총 48명의 자기공명영상이 포함되었다(평균 연령: 61.6세, 연령 분포: 10 87세).

원발 종양은 폐암 $(n=10)$, 전립선암 $(n=10)$, 유방암 $(n=6)$, 위장관계 기원의 악성 종양 $(n=4)$, 두개 내의 악성 종양 $(n=4)$, 신장암 $(n=2)$, 림프종 $(n=2)$, 요관암 $(n=2)$, 비인두암 $(n=2)$, 간암 $(n=1)$, 방광암 $(n=1)$, 담낭암 $(n=1)$, 담관암 $(n=1)$, 난소암 $(n=1)$, 후두암 $(n=1)$ 이었다. 
Fig. 1. Flow chart for patient selection.

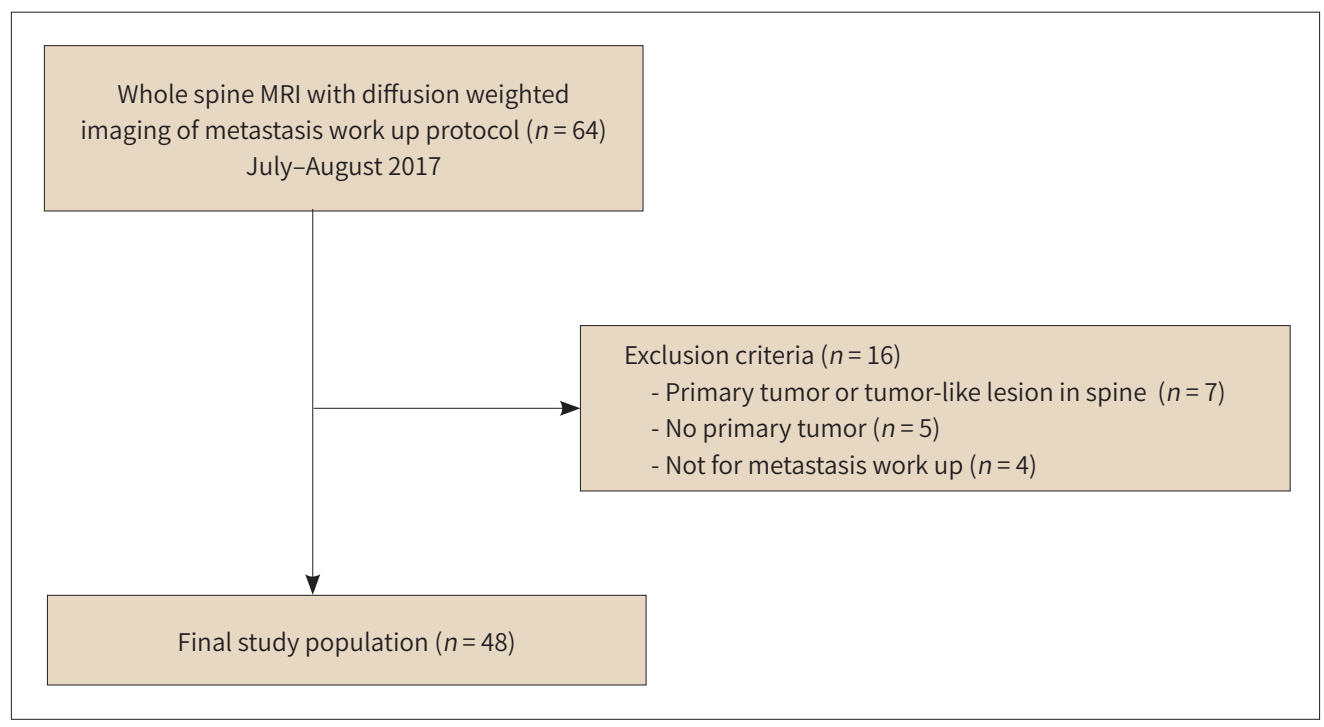

\section{자기공명영상 프로토콜}

연구 대상 환자 48명의 영상은 3.0-Tesla 자기공명영상 촬영기(Ingenia, Philips Healthcare, Best, the Netherlands)로 획득하였다. 척추 전이 여부 평가를 위한 기존의 전 척추(whole spine) $\mathrm{MRI}$ 프로토콜(conventional MRI)은 다음과 같다: $\mathrm{T} 1$ 강조 시상면 터보 스핀 에코[T1-weighted sagittal turbo-spin-echo (이하 TSE)] [repetition time (이하 TR)/echo time (이하 TE), 400 650/10 msec; slice thickness, $4 \mathrm{~mm}$; slice gap, $0.4 \mathrm{~mm}$; field of view, 25 40 cm; matrix, $440 \times 429$ ], T2 강조 축상면 터보 스핀 에코(T2-weighted sagittal TSE) (TR/TE, 3400 4400/120 msec; slice thickness, $8 \mathrm{~mm}$; slice gap, $2 \mathrm{~mm}$; field of view, 25 40 cm; matrix, $440 \times 413$ ), 그 리고 T1 강조 축상면 터보 스핀 에코(T1-weighted axial TSE) (TR/TE, 400 650/10 msec; slice thickness, $8 \mathrm{~mm}$; slice gap, $2 \mathrm{~mm}$; field of view, 25 40 cm; matrix, $440 \times 429$ ).

조영 증강 영상은 지방억제 T1 강조 터보 스핀 에코(T1-weighted multiecho dixon TSE)영상 을 얻었고, 시상면(TR/TE, 450 650/15 msec; slice thickness, 4 mm; slice gap, $0.4 \mathrm{~mm}$; field of view, 25 40 cm; matrix, $352 \times$ 391; mean acquisition time, 6 minutes 47 seconds) 및 축상면 (TR/TE, 400 650/15 msec; slice thickness, 8 mm; slice gap, 2 mm; field of view, 15 cm; matrix, $304 \times 260$; mean acquisition time, 6 minutes 54 seconds)에 대해 얻었다. 조영제는 0.1 $\mathrm{mmoL} / \mathrm{kg}$ Gd-DPTA (Magnevist; Schering, Berlin, Germany)로 정맥 주사하였다.

확산강조영상은 다발에코평면영상(multi-shot echo planar imaging)을 시상면에 대해 얻었다 (TR/TE, 2255/62 84 msec; slice thickness, 4 mm; slice gap, 0.4 mm; field of view, 25 37 cm; matrix, $148 \times 97 ; \mathrm{b}=1000 \mathrm{~s} / \mathrm{mm}^{2}$; mean acquisition time, 4 minutes 1 seconds).

\section{영상소견 분석}

3 명의 영상의학과 의사(reader 1 , 척추 영상 분야에서 17 년간의 경력을 가진 영상의학 전문의, reader 2 , 척추 영상에서 7년간의 경험을 가진 영상의학 전문의, reader 3 , 척추 영상에서 9 개월의 
경험을 가진 영상의학 전공의)가 $\mathrm{MRI}$ 를 후향적으로 검토했다. 각각의 영상의학과 의사들은 $\mathrm{MRI}$ 를 독립적으로 해석하였고, 척추 전이 평가를 위한 MRI라는 정보만 제공받았다.

각각의 영상의학과 의사는 각 환자의 척추 자기공명영상을 2 회 판독하였다. 2 회의 판독은 2 개의 다른 MRI 프로토콜에 대한 평가를 반영하였다; 첫째, 기존의 MRI 프로토콜(conventional, T1 및 $\mathrm{T} 2$ 강조 영상과 조영 증강 영상), 둘째, conventional에 DWI를 추가한 프로토콜. 동일한 환자에 대한 2 회의 판독 세션은 2 주 이상의 시간 간격을 두고 진행하여 리콜 편차(recall bias)를 줄였다. 두 번째 세션에서는 첫 번째 세션의 판독 보고서를 검토하거나 참고하지 않았다.

2회의 판독 세션에서 모두 척추 전이 여부에 대한 평가는 척추 분절(spinal segment)의 수로 기 록되었고, 각각의 분절에 대해 신뢰등급(confidence rating)을 3개의 등급으로 나누어 점수화하 여 기술하였다: 비교적 분명하게 전이가 의심되는 경우(probably metastasis: 2), 전이 여부가 애 매한 경우(doubtful: 1), 비교적 분명히 전이가 없을 것으로 판단되는 경우(probably benign: 0). 하나의 척추 분절(spinal segment)은 전방에 위치한 척추뼈몸통(vertebral body)과 척추뒤고리 (posterior element)로 나누어 평가하였다.

2회의 판독 세션에서의 영상 분석은 선행 연구에서 척추 전이를 시사하는 것으로 보았던 소견 을 근거로, 척추 전이 여부의 판단에 있어 최대한 실제 판독에 가깝게 수행하였다. 첫 번째 판독 세 션에서는 1) T1 강조 영상에서 병변이 디스크를 기준으로 그보다 낮은 신호 강도를 보일 경우, 2) 모 든 영상에서 병변 내부의 해면뼈(trabecular bone)가 소실되었을 경우, 그리고 3) 조영 증강 영상 에서 강한 조영 증강을 보일 경우의 세 가지 기준을 원칙으로 하여 비교적 분명하게 전이가 의심 되는 경우(probably metastasis: 2)로 판독하였다(16-20). 또한, T2 강조 영상에서 달무리 징후 (halo sign)가 보이는 경우, 모든 영상에서 척추 주위 조직으로의 침윤이 보이는 경우에는 전이를 의심할 수 있는 소견으로 참고하였다.

앞서 언급한 소견들이 보이지 않거나, 반대되는 소견을 보일 경우 비교적 분명히 전이가 없을 것으로 판단되는 경우(probably benign: 0)로 판독하였으며, 이러한 소견들이 모호할 경우 전이 여부가 애매한 경우(doubtful: 1)로 판독하였다.

두 번째 판독 세션에서는 첫 번째 판독 세션에서의 기준을 동일하게 적용하였고, 이에 더하여 병변이 낮은 apparent diffusion coefficient 값을 보이며, 확산 제한(diffusion restriction)을 보 일 경우 전이를 의심할 수 있는 소견으로 참고하였다(13, 15). 영상의학과 의사(reader 3)는 자기 공명영상 촬영 시점으로부터 6개월 이내에 이루어진 척추의 단순촬영(plain radiograph)과 컴퓨 터단층촬영 영상(이하 CT)에 기반하여 척추 전이의 유형을 평가했다. 척추 전이의 유형은 골용해 성(osteolytic), 골형성성(osteoblastic), 복합성(mixed)으로 구분하였다(21).

영상의학과 의사(reader 3)는 DWI의 질을 평가하였는데, DWI에서 허상에 의해 가려지는 척추 분절의 수를 해부학적 높이[vertebral level: 경추(C), 흥추(T), 요추(L), 천추(S)]에 따라 나누어 분 석하였다.

\section{통계적 분석}

데이터 분석과 관련하여 통계 학자(통계에서 15 년간의 경력을 가진 의료 통계 교수)와 상담하였다. 


\begin{tabular}{|c|c|c|c|c|c|c|c|c|c|c|c|c|c|c|}
\hline \multirow{2}{*}{ Vertebral body } & \multicolumn{13}{|c|}{ Presumed Metastatic Lesion } & \multirow{2}{*}{$\begin{array}{c}\text { Presumed } \\
\text { Non-Metastatic } \\
\text { Lesion }\end{array}$} \\
\hline & \multicolumn{7}{|c|}{ Definite Useful } & \multicolumn{5}{|c|}{ Equivocal } & $\begin{array}{c}\text { Not } \\
\text { Useful }\end{array}$ & \\
\hline Category* & 1 & 2 & 3 & 4 & 5 & 6 & 7 & 8 & 9 & 10 & 11 & 12 & 13 & 14 \\
\hline Probably metastasis & $\downarrow$ & $\downarrow$ & - & $\uparrow$ & $\uparrow$ & $\uparrow$ & $\uparrow$ & $\downarrow$ & $\downarrow$ & $\downarrow$ & - & $\uparrow$ & - & \\
\hline Doubtful & - & $\downarrow$ & $\downarrow$ & $\downarrow$ & - & $\downarrow$ & $\downarrow$ & $\uparrow$ & $\uparrow$ & $\uparrow$ & $\uparrow$ & $\uparrow$ & - & \\
\hline Probably benign & $\uparrow$ & $\uparrow$ & $\uparrow$ & - & $\downarrow$ & $\downarrow$ & $\uparrow$ & - & $\uparrow$ & $\downarrow$ & $\downarrow$ & $\downarrow$ & - & \\
\hline \multicolumn{15}{|l|}{ Reader 1} \\
\hline Number of patients & 1 & 0 & 7 & 2 & 4 & 3 & 1 & 1 & 0 & 0 & 3 & 1 & 8 & 17 \\
\hline Total & & & & 18 & & & & & & 5 & & & 8 & 17 \\
\hline \multicolumn{15}{|l|}{ Reader 2} \\
\hline Number of patients & 4 & 1 & 5 & 2 & 4 & 3 & 0 & 1 & 2 & 0 & 2 & 0 & 8 & 16 \\
\hline Total & & & & 19 & & & & & & 5 & & & 8 & 16 \\
\hline \multicolumn{15}{|l|}{ Reader 3} \\
\hline Number of patients & 0 & 1 & 7 & 2 & 3 & 2 & 1 & 3 & 0 & 0 & 2 & 0 & 10 & 17 \\
\hline Total & & & & 16 & & & & & & 5 & & & 10 & 17 \\
\hline \multicolumn{15}{|l|}{ Posterior element } \\
\hline Category ${ }^{\star}$ & 1 & 2 & 3 & 4 & 5 & 6 & 7 & 8 & 9 & 10 & 11 & 12 & 13 & 14 \\
\hline Probably metastasis & $\downarrow$ & $\downarrow$ & - & $\uparrow$ & $\uparrow$ & $\uparrow$ & $\uparrow$ & $\downarrow$ & $\downarrow$ & $\downarrow$ & - & $\uparrow$ & - & \\
\hline Doubtful & - & $\downarrow$ & $\downarrow$ & $\downarrow$ & - & $\downarrow$ & $\downarrow$ & $\uparrow$ & $\uparrow$ & $\uparrow$ & $\uparrow$ & $\uparrow$ & - & \\
\hline Probably benign & $\uparrow$ & $\uparrow$ & $\uparrow$ & - & $\downarrow$ & $\downarrow$ & $\uparrow$ & - & $\uparrow$ & $\downarrow$ & $\downarrow$ & $\downarrow$ & - & \\
\hline \multicolumn{15}{|l|}{ Reader 1} \\
\hline Number of patients & 1 & 0 & 1 & 0 & 10 & 0 & 0 & 0 & 0 & 0 & 1 & 0 & 5 & 30 \\
\hline Total & & & & 12 & & & & & & 1 & & & 5 & 30 \\
\hline \multicolumn{15}{|l|}{ Reader 2} \\
\hline Number of patients & 3 & 0 & 4 & 0 & 4 & 1 & 1 & 0 & 0 & 0 & 0 & 1 & 9 & 25 \\
\hline Total & & & & 13 & & & & & & 1 & & & 9 & 25 \\
\hline \multicolumn{15}{|l|}{ Reader 3} \\
\hline Number of patients & 0 & 2 & 2 & 0 & 4 & 1 & 0 & 0 & 0 & 1 & 2 & 1 & 10 & 25 \\
\hline Total & & & & 9 & & & & & & 4 & & & 10 & 25 \\
\hline
\end{tabular}

Data on the number of subjects.

*The patients were divided in 14 categories based on the total number of changes in the confidence rating (probable metastatic, equivocal, and probable benign segments) between sessions 1 (conventional, T1- and T2-weighted image, and contrast-enhanced MRI) and 2 (additional DW-MRI after conventional imaging) as follows: Category 1, decreased number of probable metastatic segments, no change in equivocal segments, or increased number of probable benign segments; Category 2, decreased number of probable metastatic segments, decreased number of equivocal segments, or increased number of probable benign segments; Category 3, no change of probable metastatic segments, decreased number of equivocal segments, or increased number of probable benign segments; Category 4, increased number of probable metastatic segments, decreased number of equivocal segments, or no change in probable benign segments; Category 5, increased number of probable metastatic segments, no change in equivocal segments, or decreased number of probable benign segments; Category 6, increased number of probable metastatic segments, decreased number of equivocal segments, or decreased number of probable benign segments; Category 7 , increased number of probable metastatic segments, decreased number of equivocal segments, or increased number of probable benign segments; Category 8, decreased number of probable metastatic segments, increased number of equivocal segments, or no change in probable benign segments; Category 9, decreased number of probable metastatic segments, increased number of equivocal segments, or increased number of probable benign segments; Category 10, decreased number of probable metastatic segments, increased number of equivocal segments, or decreased number of probable benign segments; Category 11, no change in probable metastatic segments, increased number of equivocal segments, or decreased number of probable benign segments; Category 12, increased number of probable metastatic segments, increased number of equivocal segments, or decreased number of probable benign segments; Category 13 , no change in probable metastatic segments, no change in equivocal segments, and no change in probable benign segments; and Category 14, all segments are probably benign in both the sessions. DW-MRI was useful in categories 1, 2, 3, 4, 5, 6, and 7. The use of DW-MRI was equivocal in categories 8 , 9 , 10, 11, and 12. DW-MRI was not useful in Category 13. No metastasis was presumed in Category 14.

DW-MRI = diffusion-weighted MRI 
모든 통계 분석은 R software package (version 2.14.2; R Foundation for Statistical Computing, Vienna, Austria) 및 Stata 14.0 (StataCorp, College Station, TX, USA)을 사용하여 수행하였다.

환자들을 첫 번째와 두 번째 판독 세션에서 척추 전이 의심 병변에 대한 신뢰등급의 변화를 기 반으로 14 개의 군(category)으로 분류하였다. 이에 대한 설명은 Table 1에 자세히 기술하였다. 예 를 들어, 1 군은 probably metastasis로 평가된 분절의 수가 감소하였고, doubtful은 변화가 없으 며, probably benign으로 평가된 분절의 수는 증가한 경우에 해당한다; 8군은 probably metastasis로 평가된 분절의 수가 감소하였고, doubtful은 증가하였으며, probably benign으로 평가된 분절의 수는 변화가 없는 경우에 해당한다.

이러한 14 개의 군을 유용한 정도(degree of usefulness)에 따라 4개의 그룹(group)으로 통합하 였다.

1) 명확히 유용한 그룹(definitely useful group), 전이의 감별에 유용한 1, 2, 3, 4, 7군과 전이의 발견에 유용한 5 군, 전이의 발견과 감별 모두에 유용한 6 군

2) 유용성이 모호한 그룹(equivocal group), 8, 9, 10, 11, 12군

3) 유용하지 않은 그룹(not useful group), 13 군

4) 척추 전이가 없는 것으로 평가된 그룹(presumed non-metastatic group), 14군

2 회의 판독 세션에서 모두 척추의 모든 분절에 대해 비교적 분명히 전이가 없을 것으로 판단되 는 경우(probably benign: 0)로 판독한 경우는 14군으로 분류하였고 신뢰등급의 변화를 평가하지 않았다. 이러한 경우에 수치상으로는 13군으로 분류되지만, 확산강조영상의 유용성을 반영하기 어렵다고 판단하였기 때문이다.

환자의 임상 정보(나이, 성별, 원발 종양, 척추 전이의 유형)를 Fisher's exact test로 분석하였고, 유용성을 반영한 3개의 그룹과 첫 번째 세션에서의 probably metastasis로 평가한 분절의 수의 상관관계를 kruskal wallis test로 평가하였다.

유용성을 반영한 3개의 그룹과 해부학적 위치(척추뼈몸통, 척추뒤고리)의 상관관계를 Fisher's

Fig. 2. Graphs of the usefulness of diffusion-weighted MRI depending on the anatomic position (vertebral body; A and posterior element; B), evaluated by each reader (reader 1 , reader 2 , and reader 3 ).

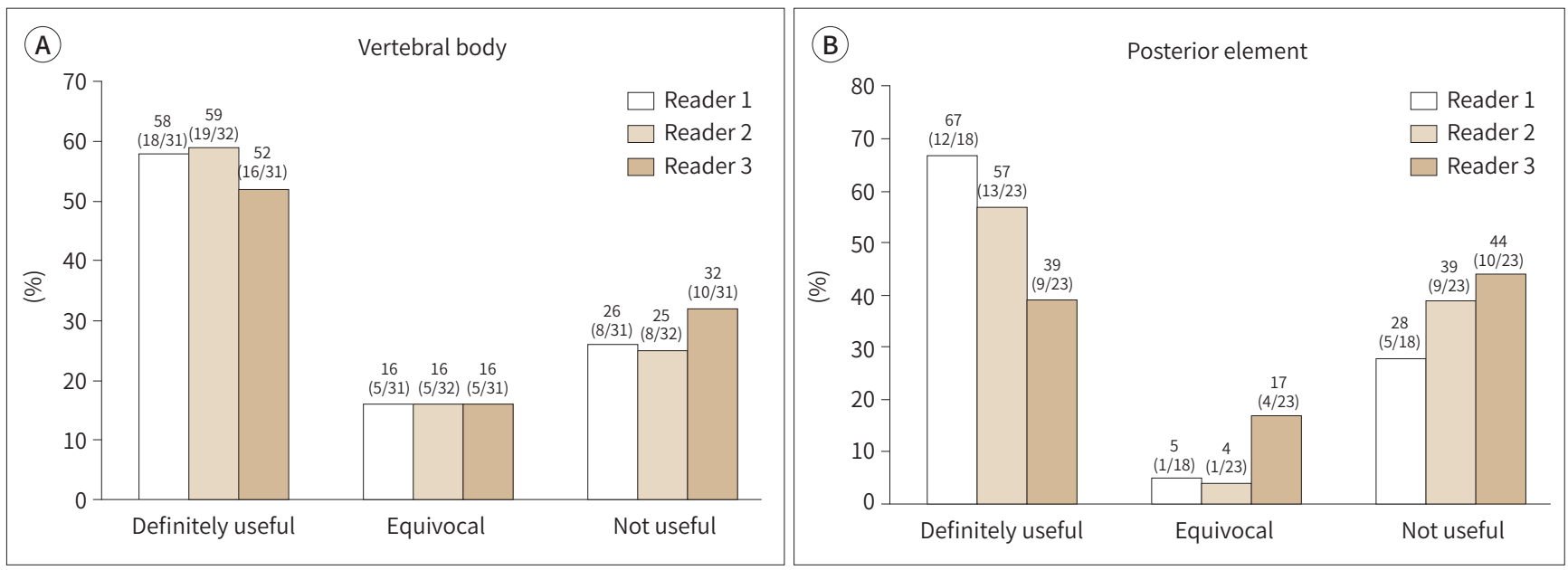


Table 2. Usefulness of Diffusion-Weighted MRI Depending on the Clinical Information and Number of Involved Segments on Conventional MRI at Each Anatomic Location

\begin{tabular}{|c|c|c|c|c|c|}
\hline & \multicolumn{3}{|c|}{ Presumed Metastatic Lesion } & \multirow{2}{*}{$p$-Value } & \multirow{2}{*}{ Presumed Non-Metastatic Lesion } \\
\hline & Definite Useful & Equivocal & Not Useful & & \\
\hline \multicolumn{6}{|l|}{ Vertebral body } \\
\hline \multicolumn{6}{|l|}{ Reader 1} \\
\hline Age $^{\star}$ & $59.4 \pm 17.2$ & $66.2 \pm 9.4$ & $58.3 \pm 7.9$ & 0.064 & \\
\hline Sex & & & & 0.59 & \\
\hline Male & 10 & 4 & 6 & & \\
\hline Female & 8 & 1 & 2 & & \\
\hline Primary cancer & & & & 0.822 & \\
\hline Lung & 3 & 1 & 2 & & \\
\hline Prostate & 2 & 2 & 1 & & \\
\hline Breast & 4 & 0 & 2 & & \\
\hline Miscellaneous $^{\dagger}$ & 9 & 2 & 3 & & \\
\hline Type & & & & 0.517 & \\
\hline Pure lytic & 3 & 1 & 4 & & \\
\hline Blastic and mixed & 6 & 1 & 2 & & \\
\hline Unknown ${ }^{\ddagger}$ & 9 & 3 & 2 & & \\
\hline Number of segment on session $1^{*}$ & $5.8 \pm 8.2$ & $7.8 \pm 11.6$ & $10.2 \pm 13.3$ & 0.278 & \\
\hline Total & 18 & 5 & 8 & & 17 \\
\hline \multicolumn{6}{|l|}{ Reader 2} \\
\hline Age $^{\star}$ & $66.2 \pm 12.0$ & $56.6 \pm 11.1$ & $61.6 \pm 9.8$ & 0.829 & \\
\hline Sex & & & & 0.875 & \\
\hline Male & 11 & 3 & 6 & & \\
\hline Female & 8 & 2 & 2 & & \\
\hline Primary cancer & & & & 0.953 & \\
\hline Lung & 4 & 0 & 2 & & \\
\hline Prostate & 5 & 1 & 1 & & \\
\hline Breast & 7 & 3 & 3 & & \\
\hline Miscellaneous $^{\dagger}$ & 19 & 5 & 8 & & \\
\hline Type & & & & 0.597 & \\
\hline Pure lytic & 3 & 2 & 3 & & \\
\hline Blastic and mixed & 6 & 2 & 2 & & \\
\hline Unknown ${ }^{\ddagger}$ & 10 & 1 & 3 & & \\
\hline Number of segment on session $1^{\star}$ & $5.9 \pm 9.6$ & $18.2 \pm 14.9$ & $8.2 \pm 12.9$ & 0.423 & \\
\hline Total & 19 & 5 & 8 & & 16 \\
\hline \multicolumn{6}{|l|}{ Reader 3} \\
\hline Age $^{\star}$ & $66.5 \pm 11.0$ & $66.5 \pm 15.1$ & $54.1 \pm 11.1$ & 0.764 & \\
\hline Sex & & & & 0.725 & \\
\hline Male & 6 & 3 & 5 & & \\
\hline Female & 5 & 1 & 2 & & \\
\hline Primary cancer & & & & 0.115 & \\
\hline Lung & 3 & 0 & 1 & & \\
\hline Prostate & 1 & 3 & 0 & & \\
\hline
\end{tabular}


Table 2. Usefulness of Diffusion-Weighted MRI Depending on the Clinical Information and Number of Involved Segments on Conventional MRI at Each Anatomic Location (Continued)

\begin{tabular}{|c|c|c|c|c|c|}
\hline & \multicolumn{3}{|c|}{ Presumed Metastatic Lesion } & \multirow{2}{*}{$p$-Value } & \multirow{2}{*}{ Presumed Non-Metastatic Lesion } \\
\hline & Definite Useful & Equivocal & Not Useful & & \\
\hline Breast & 2 & 1 & 2 & & \\
\hline Miscellaneous $^{\dagger}$ & 5 & 0 & 4 & & \\
\hline Type & & & & 0.912 & \\
\hline Pure lytic & 2 & 1 & 3 & & \\
\hline Blastic and mixed & 5 & 2 & 3 & & \\
\hline Unknown ${ }^{\ddagger}$ & 4 & 1 & 1 & & \\
\hline Number of segment on session $1^{*}$ & $4.2 \pm 6.1$ & $1.4 \pm 1.7$ & $17.6 \pm 14.7$ & $<0.001$ & \\
\hline Total & 11 & 4 & 7 & & 26 \\
\hline \multicolumn{6}{|l|}{ Posterior element } \\
\hline \multicolumn{6}{|l|}{ Reader 1} \\
\hline Age* $^{\star}$ & $59.8 \pm 10.5$ & $66 \pm 0$ & $59.6 \pm 13.9$ & 0.501 & \\
\hline Sex & & & & 0.387 & \\
\hline Male & 9 & 0 & 3 & & \\
\hline Female & 3 & 1 & 2 & & \\
\hline Primary cancer & & & & 1 & \\
\hline Lung & 3 & 0 & 1 & & \\
\hline Prostate & 2 & 0 & 0 & & \\
\hline Breast & 2 & 0 & 1 & & \\
\hline Miscellaneous $^{\dagger}$ & 5 & 1 & 3 & & \\
\hline Type & & & & 0.378 & \\
\hline Pure lytic & 5 & 0 & 2 & & \\
\hline Blastic and mixed & 4 & 0 & 3 & & \\
\hline Unknown ${ }^{\neq}$ & 3 & 1 & 0 & & \\
\hline Number of segment on session $1^{\star}$ & $5.7 \pm 5.5$ & $0 \pm 0$ & $14.8 \pm 12.6$ & 0.039 & \\
\hline Total & 12 & 1 & 5 & & 30 \\
\hline \multicolumn{6}{|l|}{ Reader 2} \\
\hline Age* $^{\star}$ & $63.2 \pm 12.0$ & $55 \pm 0$ & $61.2 \pm 12.0$ & 0.986 & \\
\hline Sex & & & & 0.029 & \\
\hline Male & 5 & 1 & 8 & & \\
\hline Female & 8 & 0 & 1 & & \\
\hline Primary cancer & & & & 0.257 & \\
\hline Lung & 3 & 1 & 1 & & \\
\hline Prostate & 3 & 0 & 1 & & \\
\hline Breast & 4 & 0 & 1 & & \\
\hline Miscellaneous $^{\dagger}$ & 3 & 0 & 6 & & \\
\hline Type & & & & 0.448 & \\
\hline Pure lytic & 2 & 1 & 3 & & \\
\hline Blastic and mixed & 7 & 0 & 3 & & \\
\hline Unknown $^{\ddagger}$ & 4 & 0 & 3 & & \\
\hline Number of segment on session $1^{\star}$ & $4.5 \pm 6.9$ & $7 \pm 0$ & $14.2 \pm 11.6$ & 0.114 & \\
\hline Total & 13 & 1 & 9 & & 25 \\
\hline
\end{tabular}


exact test로 분석하였다.

모든 검사에서, 통계적으로 유의한 차이를 나타내는 지표로 $p$-value를 사용하였고, $p<0.05$ 를 기준으로 하였다.

Table 2. Usefulness of Diffusion-Weighted MRI Depending on the Clinical Information and Number of Involved Segments on Conventional MRI at Each Anatomic Location (Continued)

\begin{tabular}{|c|c|c|c|c|c|}
\hline & \multicolumn{3}{|c|}{ Presumed Metastatic Lesion } & \multirow{2}{*}{$p$-Value } & \multirow{2}{*}{ Presumed Non-Metastatic Lesion } \\
\hline & Definite useful & Equivocal & Not Useful & & \\
\hline \multicolumn{6}{|l|}{ Reader 3} \\
\hline Age* $^{*}$ & $67.4 \pm 12.3$ & $61 \pm 11.0$ & $59.3 \pm 12.8$ & 0.959 & \\
\hline Sex & & & & 0.336 & \\
\hline Male & 4 & 2 & 8 & & \\
\hline Female & 5 & 2 & 2 & & \\
\hline Primary cancer & & & & 0.183 & \\
\hline Lung & 3 & 0 & 1 & & \\
\hline Prostate & 1 & 2 & 1 & & \\
\hline Breast & 1 & 2 & 2 & & \\
\hline Miscellaneous $^{\dagger}$ & 4 & 0 & 6 & & \\
\hline Type & & & & 0.756 & \\
\hline Pure lytic & 1 & 1 & 4 & & \\
\hline Blastic and mixed & 5 & 2 & 3 & & \\
\hline Unknown ${ }^{\ddagger}$ & 3 & 1 & 3 & & \\
\hline Number of segment on session $1^{\star}$ & $2.3 \pm 3.4$ & $2.2 \pm 2.9$ & $15.2 \pm 11.4$ & 0.002 & \\
\hline Total & 9 & 4 & 10 & & 25 \\
\hline
\end{tabular}

Data on the number of subjects, except when mentioned otherwise.

*Data are expressed as mean \pm standard deviation, with the ranges enclosed in parentheses.

† Primary cancers, except lung and prostate or breast cancers, are classified as miscellaneous.

${ }^{\ddagger}$ Cases of no possible radiographic or CT scan within 6 months from the MRI to evaluate the metastatic type are classified as unknown.

Table 3. Usefulness of Diffusion-Weighted MRI Depending on the Anatomic Location

\begin{tabular}{|c|c|c|c|c|c|}
\hline & \multicolumn{3}{|c|}{ Presumed Metastatic Lesion } & \multirow{2}{*}{$p$-Value } & \multirow{2}{*}{ Presumed Non-Metastatic Lesior } \\
\hline & Definitely Useful & Equivocal & Not Useful & & \\
\hline \multicolumn{6}{|l|}{ Reader 1} \\
\hline Position & & & & 0.55 & \\
\hline Vertebral body & 18 & 5 & 8 & & 17 \\
\hline Posterior element ${ }^{\star}$ & 12 & 1 & 5 & & 30 \\
\hline \multicolumn{6}{|l|}{ Reader 2} \\
\hline Position & & & & 0.29 & \\
\hline Vertebral body & 19 & 5 & 8 & & 16 \\
\hline Posterior element ${ }^{\star}$ & 13 & 1 & 9 & & 25 \\
\hline \multicolumn{6}{|l|}{ Reader 3} \\
\hline Position & & & & 0.64 & \\
\hline Vertebral body & 16 & 5 & 10 & & 17 \\
\hline Posterior element ${ }^{\star}$ & 9 & 4 & 10 & & 25 \\
\hline
\end{tabular}

Data on the number of subjects.

*The sacrum was evaluated only in the vertebral body because it is difficult to count the number of involved segments in the posterior element. 
분석 결과는 Table 1과 Fig. 2에 요약하여 정리하였다.

48 명의 연구 대상 환자들 중 척추뼈몸통을 기준으로 하였을 때, reader 1 의 판독상에서 definitely useful은 18명, equivocal은 5명, not useful은 8명이었고, presumed non-metastatic lesion은 17 명이었다. Reader 2 의 판독상에서는 definitely useful은 19명, equivocal은 5명, not useful은 8명이었고, presumed non-metastatic lesion은 16 명이었다. Reader 3 의 판독상에서는 definitely useful은 16명, equivocal은 5명, not useful은 10명이었고, presumed non-metastatic lesion은 17명이었다. Presumed non-metastatic lesion을 포함하지 않고 계산하였을 때, definitely useful 그룹은 reader 1에서 58\%(18/31), reader 2에서 59\%(19/32), reader 3에서 52\%(16/31)였다.

48명의 연구 대상 환자들 중 척추뒤고리를 기준으로 하였을 때, reader 1 의 판독상에서 definitely useful은 12명, equivocal은 1명, not useful은 5명이었고, presumed non-metastatic lesion은 30명이었다. Reader 2의 판독상에서는 definitely useful은 13명, equivocal은 1명, not useful은 9명이었고, presumed non-metastatic lesion은 25명이었다. Reader 3의 판독상에서는 definitely useful은 9명, equivocal은 4명, not useful은 10명이었고, presumed non-metastatic lesion은 25명이었다. Presumed non-metastatic lesion을 포함하지 않고 계산하였을 때, definitely useful 그룹은 reader 1에서 67\%(12/18), reader 2에서 57\%(13/23), reader 3에서 39\% $(9 / 23)$ 였다.

Reader 3의 판독에 한하여, 첫 번째 세션에서 probably metastasis로 평가된 분절의 수는 척추 뼈몸통과 척추뒤고리를 기준으로 하였을 때 모두 definitely useful, equivocal 그리고 not useful group 사이에 유의한 차이를 보였다[척추뼈몸통 $(p<0.001)$, 척추뒤고리 $(p=0.002)]$. Reader 1 의 경우, 척추뒤고리로 분석하였을 때, 유의한 차이를 보였다 $(p=0.039)$. Reader 2에서는 모든 경우 에서 유의한 차이가 없었다.

모든 reader의 판독에서 연령, 성별, 원발 종양, 전이의 유형 및 해부학적 위치(척추뼈몸통, 척추

Table 4. Quality of DW-MRI

\begin{tabular}{lcccc}
\hline \multicolumn{1}{c}{ Category $^{*}$} & Poor & Fair & Good & Excellent \\
\hline DWI image quality & & & & \\
Number of patients & 7 & 10 & 15 & 16 \\
DWl image quality by vertebral level & & & 2 & 40 \\
C & 0 & 6 & 13 & 23 \\
T & 6 & 6 & 0 & 48 \\
L & 0 & 0 & 0 & 48 \\
S & 0 & 0 & & \\
\hline
\end{tabular}

Data on the number of subjects.

*The patients were divided in 4 grades based on the number of spinal segments not visible on DW-MRI because of artifacts: poor (0), more than 5 segments are not visible; fair (1), 3-4 segments are not visible; good (2), 1-2 segments are not visible; excellent (3), all the spinal segments are visible.

DW-MRI = diffusion-weighted MRI, DWI = diffusion weighted image 
뒤고리)는 reader 2 의 판독에 한하여 척추뒤고리로 분석하였을 때, 성별에서 세 군 간에 유의한 차 이가 있었고, 이외에 나머지 경우에는 세 군 간에 유의한 차이는 없었다(Tables 2, 3).

DWI에서 허상에 의해 가려지는 척추 분절의 수를 vertebral level 별로 분석한 결과는 Table 4 에 요약하였다. 요추와 천추의 경우 가려지는 경우가 없었고, 경추와 흥추 중에서는 흥추가 가려 지는 경우가 더 많았으며 영상의 질이 poor인 환자는 흥추가 가려지는 환자 6명이었다(Fig. 3).

\section{고찰}

본 연구에서 확산강조 자기공명영상을 추가하였을 때 definitely useful로 평가된 경우는 척추 뼈몸통 기준으로 52 59\%였고, 척추뒤고리 기준으로 39 67\%였다. 확산강조 자기공명영상이 척 추 전이의 발견과 감별(characterization) 모두에 있어서 비교적 유용하다는 것을 보여준 결과이 다. Definitely useful, equivocal, not useful 그룹 사이에서 3명의 reader에서 모두 유의한 차이 를 보이는 임상적 요인 혹은 영상의학적 요인은 없었다.

우리는 척추뒤고리를 기준으로 하였을 때 척추뼈몸통을 기준으로 하였을 때보다 유용할 것으로 예상하였지만, 척추뼈몸통과 척추뒤고리를 기준으로 하였을 때, 유용한 정도에 유의한 차이가 없 었다. 그러나, Fig. 4에 기술한 환자 1 명은 첫 번째 판독 세션에서 세 명의 reader가 모두 T3 척추 분절의 척추뒤고리에 위치한 작은 병변을 발견하지 못하였지만, 두 번째 판독 세션에서 확산 제한 을 보이는 해당 병변을 발견하였다. 이 환자는 확산강조영상이 전이의 발견에 유용한 5 군(useful in detection of metastasis), 그리고 명확히 유용한 그룹(definitely useful group)으로 분류되었다.

또한, Fig. 5 에 기술한 환자 1 명은 첫 번째 판독 세션에서 세 명의 reader가 모두 L2 척추 분절의 병변에 대해서 전이 여부가 애매한 경우(doubtful)로 판독하였으나, 두 번째 판독 세션에서 해당 병변이 확산 제한을 보이지 않아 비교적 분명히 전이가 없을 것으로 판단되는 경우 probably be-

Fig. 3. Graphs of the quality of diffusion-weighted MRI.

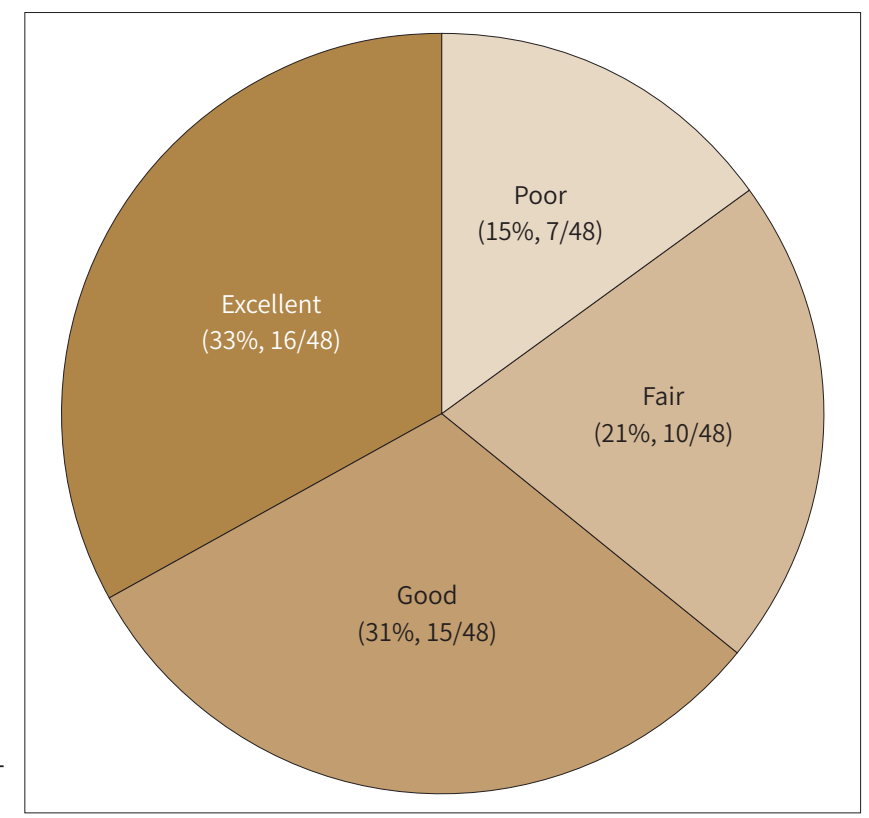


nign)로 판독하였다. 이 환자는 확산강조영상이 전이의 감별에 유용한 3군(useful in characterization of metastasis), 그리고 명확히 유용한 그룹(definitely useful group)으로 분류되었다.

요통은 척추 전이의 가장 흔한 증상이고 이로부터 수 주에서 수개월 후에는 신경학적 증상이 발 현되는 경우가 많다(22). 척추 전이 환자에게 가장 중요한 치료 목적은 통증을 감소시키고, 기능을 회복시키는 것이다(23). 척추 전이 여부 및 전이된 분절 수의 평가는 암 환자에게서 앞서 언급한 증상이 있을 때 원인에 대한 평가에 있어 매우 중요하다(24).

Fig. 4. MR images of an 82-year-old man with prostate cancer. Sagittal T1-weighted (A), sagittal T2-weighted turbo spin-echo (B), and sagittal fat-suppressed contrast-enhanced T1-weighted MR images showing probable metastasis at T7-T9 spinal segments (C). Diffusion-weighted MR images $[b=1000$ (D) and apparent diffusion coefficient map (E)] also showing probable metastasis at T7-T9 spinal segments, along with a small probable metastasis at the T3 spinal segment (arrows on D and E). Sagittal T1-weighted (A), sagittal T2-weighted turbo spin-echo (B), and sagittal fat-suppressed contrast-enhanced T1-weighted MR images showing no definite spinal metastasis at the T3 spinal segment (C).

$\mathrm{MR}=$ magnetic resonance
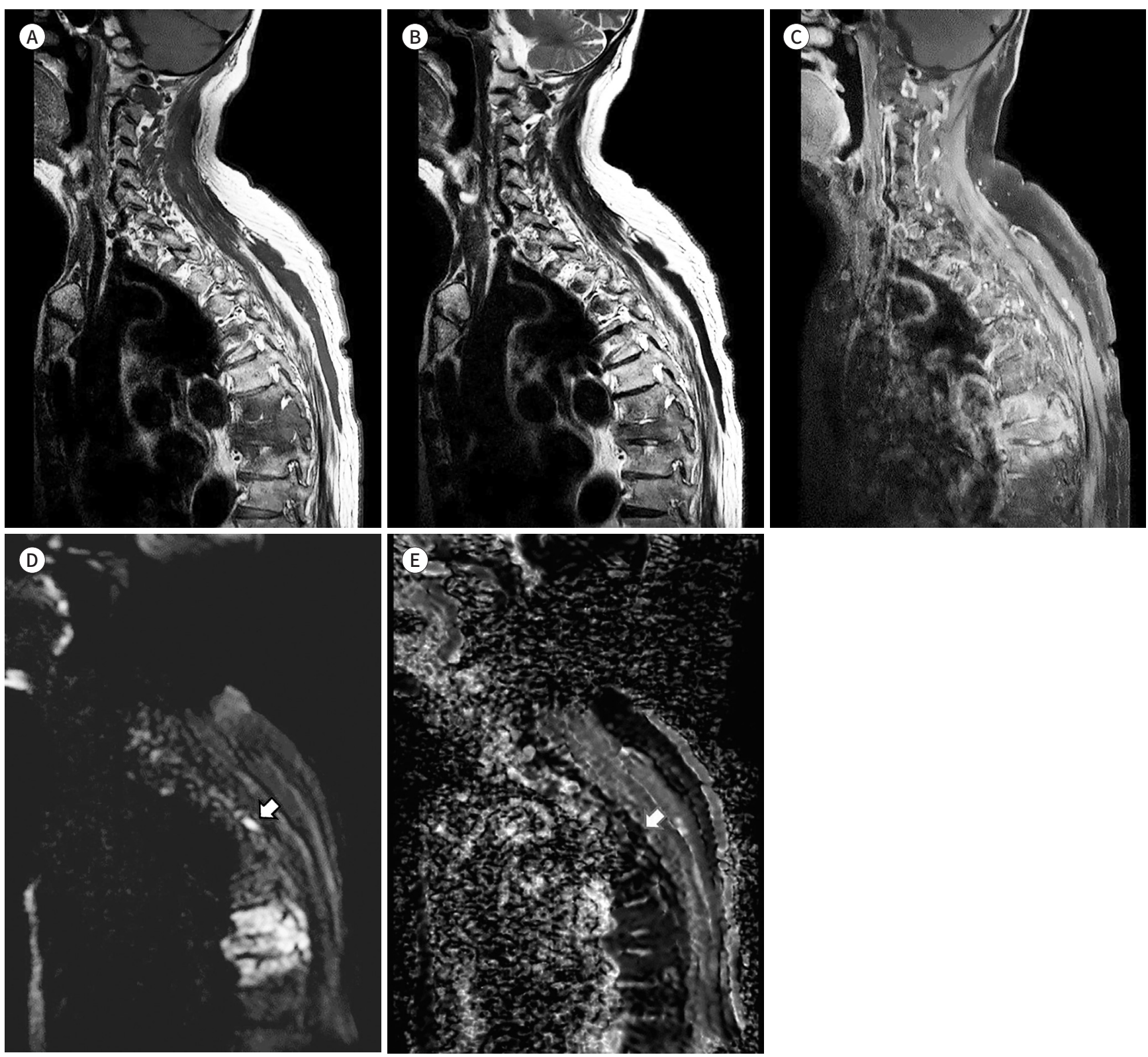
ACR에 따르면, 척추 전이 여부 및 전이된 분절 수의 평가에 있어 MRI는 현재 가장 중요한 modality로 사용되고 있다(8). 병적 압박 골절의 감별에서 유용성이 확인된 DWI를 척추 전이 여부 및 전이된 분절 수의 평가에도 적용한다면, 더욱 정확한 평가가 가능할 것이다. 이는 결국, 척추 전 이의 조기 발견 및 감별을 가능하게 할 것이고, 이를 토대로 한 수술적 혹은 방사선 치료 등의 척추 전이의 치료 계획 수립을 더욱 정교하게 하는 데 기여할 수 있을 것이다.

Fig. 5. MR images of a 66-year-old man with hepatocellular carcinoma.

A. Sagittal T1-weighted turbo spin-echo MR image showing a hypointense round nodular lesion (arrow) at the L2 vertebral body.

B. Sagittal T2-weighted MR image showing an isointense lesion (arrow) at the same location.

C-E. Sagittal fat-suppressed contrast-enhanced T1-weighted MR image showing peripheral enhancement of the L2 lesion (arrow on C). The patient's positron emission tomography-CT report showed this lesion as one of probable metastasis. Diffusion-weighted MR images [ $b=1000$ (D) and apparent diffusion coefficient map (E)] showing no diffusion restriction in the L2 lesion (arrows on D and E).

$\mathrm{MR}=$ magnetic resonance
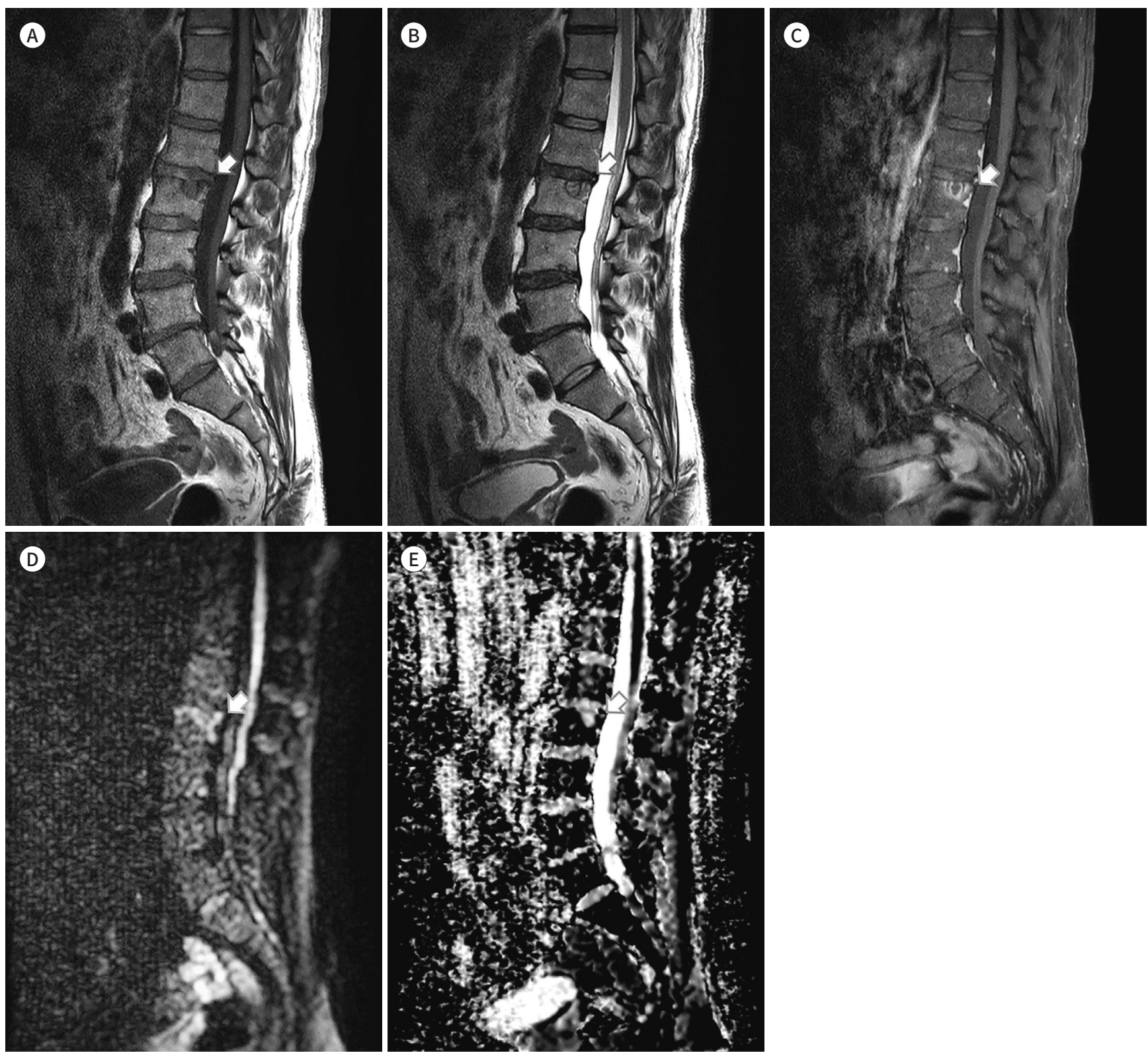
본 연구에는 몇 가지 제한점이 있다. 첫째로, 전이로 생각된 병변들이 병리학적으로 평가되지 않 았고, 영상의학적으로 추적관찰하여 비교하지 않았다. 둘째, 2회의 세션 사이의 비교 시 전체 분절 수를 기준으로 신뢰등급의 변화를 평가하였기 때문에 각 분절의 신뢰등급 변화는 충분히 반영되 지 않았을 가능성이 있다. 셋째, 연구 대상 환자의 수가 상대적으로 적었다. Definitely useful 그룹 의 대상 환자 수는 파라 메트릭 분석(parametric analysis)에 사용 가능했지만 equivocal 과 not useful group에는 사용할 수 없었다.

14 개의 군(category)으로 분류하여 분석하였을 때, 기존의 자기공명영상(conventional MRI)에 확산강조영상(DWI)을 추가한 경우가 척추 전이의 발견(detection) 및 감별(characterization)에 있어서 유용하였지만, 해부학적 위치에 따른 유용성에 차이는 없었다.

\section{Author Contributions}

Conceptualization, K.Y.J., P.C., L.J.W.; data curation, K.Y.J., P.C., L.J.W.; investigation, K.Y.J., P.C., L.J.W.; methodology, L.E., K.Y., A.J.M., K.H.S.; project administration, L.J.W.; resources, K.Y.J., L.J.W.; supervision, L.J.W.; visualization, K.Y.J.; writing-original draft, K.Y.J., L.E., L.J.W.; and writing-review \& editing, L.E., K.Y., A.J.M., K.H.S.

\section{Conflicts of Interest}

The authors have no potential conflicts of interest to disclose.

\section{REFERENCES}

1. Böhm P, Huber J. The surgical treatment of bony metastases of the spine and limbs. J Bone Joint Surg Br 2002;84:521-529

2. Witham TF, Khavkin YA, Gallia GL, Wolinsky JP, Gokaslan ZL. Surgery insight: current management of epidural spinal cord compression from metastatic spine disease. Nat Clin Pract Neurol 2006;2:87-94; quiz 116

3. Perrin RG. Metastatic tumors of the axial spine. Curr Opin Oncol 1992;4:525-532

4. Keogh C, Bergin D, Brennan D, Eustace S. MR imaging of bone tumors of the cervical spine. Magn Reson Imaging Clin N Am 2000;8:513-528

5. Avrahami E, Tadmor R, Dally O, Hadar H. Early MR demonstration of spinal metastases in patients with normal radiographs and CT and radionuclide bone scans. J Comput Assist Tomogr 1989;13:598-602

6. Algra PR, Bloem JL, Tissing H, Falke TH, Arndt JW, Verboom LJ. Detection of vertebral metastases: comparison between MR imaging and bone scintigraphy. Radiographics 1991;11:219-232

7. Smoker WR, Godersky JC, Knutzon RK, Keyes WD, Norman D, Bergman W. The role of MR imaging in evaluating metastatic spinal disease. AJR Am J Roentgenol 1987;149:1241-1248

8. Roberts CC, Daffner RH, Weissman BN, Bancroft L, Bennett DL, Blebea JS, et al. ACR appropriateness criteria on metastatic bone disease. J Am Coll Radiol 2010;7:400-409

9. Expert Panel on Radiation Oncology-Bone Metastases, Lo SS, Ryu S, Chang EL, Galanopoulos N, Jones J, et al. ACR Appropriateness Criteria ${ }^{\circledR}$ metastatic epidural spinal cord compression and recurrent spinal metastasis. J Palliat Med 2015;18:573-584

10. Patel ND, Broderick DF, Burns J, Deshmukh TK, Fries IB, Harvey HB, et al. ACR appropriateness criteria low back pain. J Am Coll Radiol 2016;13:1069-1078

11. Namimoto T, Yamashita Y, Sumi S, Tang Y, Takahashi M. Focal liver masses: characterization with diffusionweighted echo-planar MR imaging. Radiology 1997;204:739-744

12. Woodhams R, Matsunaga K, Iwabuchi K, Kan S, Hata H, Kuranami M, et al. Diffusion-weighted imaging of malignant breast tumors: the usefulness of apparent diffusion coefficient (ADC) value and ADC map for the detection of malignant breast tumors and evaluation of cancer extension. J Comput Assist Tomogr 2005; 29:644-649

13. Sung JK, Jee WH, Jung JY, Choi M, Lee SY, Kim YH, et al. Differentiation of acute osteoporotic and malignant compression fractures of the spine: use of additive qualitative and quantitative axial diffusion-weighted MR imaging to conventional MR imaging at 3.0 T. Radiology 2014;271:488-498 
14. Nakanishi K, Kobayashi M, Nakaguchi K, Kyakuno M, Hashimoto N, Onishi H, et al. Whole-body MRI for detecting metastatic bone tumor: diagnostic value of diffusion-weighted images. Magn Reson Med Sci 2007;6:147155

15. Byun WM, Shin SO, Chang Y, Lee SJ, Finsterbusch J, Frahm J. Diffusion-weighted MR imaging of metastatic disease of the spine: assessment of response to therapy. AJNR Am J Neuroradiol 2002;23:906-912

16. Daffner RH, Lupetin AR, Dash N, Deeb ZL, Sefczek RJ, Schapiro RL. MRI in the detection of malignant infiltration of bone marrow. AJR Am J Roentgenol 1986;146:353-358

17. Carroll KW, Feller JF, Tirman PF. Useful internal standards for distinguishing infiltrative marrow pathology from hematopoietic marrow at MRI. J Magn Reson Imaging 1997; 7:394-398

18. Chen YC, Sosnoski DM, Mastro AM. Breast cancer metastasis to the bone: mechanisms of bone loss. Breast Cancer Res 2010;12:215

19. Shah LM, Salzman KL. Imaging of spinal metastatic disease. Int J Surg Oncol 2011;2011:769753

20. An C, Lee YH, Kim S, Cho HW, Suh JS, Song HT. Characteristic MRI findings of spinal metastases from various primary cancers: retrospective study of pathologically-confirmed cases. Investig Magn Reson Imaging 2013;17:8-18

21. Fisher CG, DiPaola CP, Ryken TC, Bilsky MH, Shaffrey Cl, Berven SH, et al. A novel classification system for spinal instability in neoplastic disease: an evidence-based approach and expert consensus from the Spine Oncology Study Group. Spine (Phila Pa 1976) 2010;35:E1221-1229

22. Bilsky MH, Lis E, Raizer J, Lee H, Boland P. The diagnosis and treatment of metastatic spinal tumor. Oncologist 1999;4:459-469

23. Georgy BA. Metastatic spinal lesions: state-of-the-art treatment options and future trends. AJNR Am J Neuroradiol 2008;29:1605-1611

24. Jacobs WB, Perrin RG. Evaluation and treatment of spinal metastases: an overview. Neurosurg Focus 2001; 11:e10

척추 전이의 진단: 확산강조 자기공명영상 추가의 유용성

김용주 ${ }^{1} \cdot$ 이준우 ${ }^{*} \cdot$ 이영준 ${ }^{1} \cdot$ 박찬규 $^{2} \cdot$ 강유선 ${ }^{1} \cdot$ 안중모 ${ }^{1} \cdot$ 강흥식 ${ }^{1}$

목적 척추 전이의 평가에 있어 확산강조 자기공명영상의 유용성을 확인하고자 한다.

대상과 방법 2017년 7월과 8월 사이에 얻어진, 척추 외 원발암이 있는 환자들 중 척추 전이 평 가를 위한 확산강조 자기공명영상 48 건을 영상의학과 의사 3 명이 후향적으로 평가하였다. 척 추 전이 평가에 대한 확산강조영상의 유용성을 2 세션 사이의 신뢰등급 변화를 기반으로 4그 룹으로 평가하였다: 세션 1 (T1 및 T2 강조 영상과 조영 증강 영상), 세션 2 (확산강조영상을 추가). 임상 정보(나이, 성별, 원발 종양, 전이의 유형), 세션 1의 probably metastasis 분절 수, 해부학적 위치(척추뼈몸통, 척추뒤고리)와 유용성 간의 상관관계를 분석하였다.

결과 척추뼈몸통 기준으로 definitely useful 그룹은 reader 1,2 , 3에서 각각 $18,19,16$ 명, 척 추뒤고리 기준으로 $12,13,9$ 명이었다. 척추 전이가 없는 그룹을 제외하면, 확산강조영상은 척추뼈몸통 기준 52 59\%, 척추뒤고리 기준 39 67\%에서 유용하였다. 나이, 성별, 원발 종양, 전이의 유형, 해부학적 위치에 따른 유용성에 유의한 차이는 없었다.

결론 확산강조 자기공명영상은 척추 전이의 평가에 유용하였지만, 해부학적 위치에 따른 유 용성에 차이는 없었다.

${ }^{1}$ 분당서울대학교병원 영상의학과, ${ }^{2}$ 부산대학교 의과대학 양산부산대학교병원 영상의학과 